\title{
Interactive virtual optical laboratories
}

\section{Xuan Liu, Yi Yang}

Xuan Liu, Yi Yang, "Interactive virtual optical laboratories," Proc. SPIE 10452, 14th Conference on Education and Training in Optics and Photonics: ETOP 2017, 104521D (16 August 2017); doi: 10.1117/12.2268177

SDIE Event: 14th Conference on Education and Training in Optics and Photonics, ETOP 2017, 2017, Hangzhou, China 


\title{
Interactive virtual optical laboratories
}

\author{
${ }^{1}$ Xuan Liu* and ${ }^{2}$ Yi Yang \\ ${ }^{1}$ Department of Electrical and Computer Engineering, New Jersey Institute of Technology \\ Newark, NJ, 07102, USA \\ ${ }^{2}$ Department of Electrical and Computer Engineering, Penn State University, \\ Abington, PA, 19001, USA \\ *xliu@njit.edu
}

\begin{abstract}
Laboratory experiences are essential for optics education. However, college students have limited access to advanced optical equipment that is generally expensive and complicated. Hence there is a need for innovative solutions to expose students to advanced optics laboratories. Here we describe a novel approach, interactive virtual optical laboratory (IVOL) that allows unlimited number of students to participate the lab session remotely through internet, to improve laboratory education in photonics. Although students are not physically conducting the experiment, IVOL is designed to engage students, by actively involving students in the decision making process throughout the experiment.
\end{abstract}

\section{INTRODUCTION}

Laboratory experiences are essential for optics education. However, equipment involved in advanced optical laboratory is generally expensive and complicated, and college students often have limited access to advanced optical laboratory education, due to financial and resource constraint. Hence there is a need for innovative solutions to expose students to advanced optics laboratories. A virtual classroom environment enabled by matured information technology, may address this challenge and allow unlimited number of students to have virtual laboratory experience.

While distance education has been investigated for decades, the study on distance teaching of laboratory class remains quite limited [1-4]. Students learn from a laboratory class, because of the spontaneous nature of the learning process and the direct physical interaction between the learner and the learning material. As a result, it is extremely challenging to virtualized laboratory education. On the other hand, despite the physical distance between the learners (students) and the learning materials (components and equipment), virtual classroom does not exclude an educator who is constantly interacting with the learners. Therefore, the learning effectiveness can be strengthened with the instructor providing intellectual stimuli to the students. Here we describe a novel approach, interactive virtual optical laboratory (IVOL), to improve laboratory education in biophotonics.

\section{INTERACTIVE VIRTUAL OPTICAL LABORATORY}

IVOL has the following major components, as shown in Figure 1. First, there is a classroom where learning takes place. The students share the classroom with their instructor. Second, IVOL also depends on a physical laboratory environment where experiments are conducted synchronously, or asynchronously. Finally, IVOL requires IT

14th Conference on Education and Training in Optics and Photonics: ETOP 2017, edited by Xu Liu,

Xi-Cheng Zhang, Proc. of SPIE Vol. 10452, 104521D · (c) 2017 ICO, IEEE, OSA, SPIE

CCC code: $0277-786 X / 17 / \$ 18 \cdot$ doi: $10.1117 / 12.2268177$

Proc. of SPIE Vol. 10452 104521D-1 
technological support to enable the virtual classroom environment.

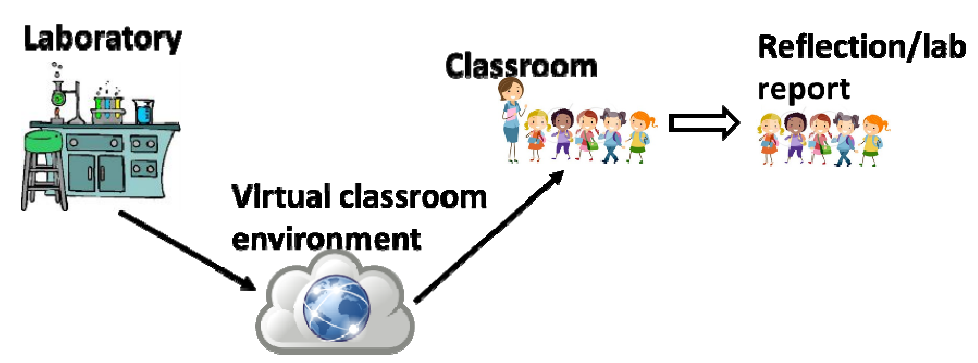

Figure. 1. IVOL consists of a classroom where learning takes place, a laboratory environment where experiments are conducted, and a virtual classroom environment.

\section{MODULES OF IVOL}

To fully take advantage of the interaction between the educator and the learner, IVOL requires learning materials that are designed specifically to simulate the actual procedure of laboratory experiment. In general, an IVOL experiment has several sub-sessions that take place sequentially, as shown in Figure 2. At the beginning of each sub-session, students are given a multiple-choice question to determine how the experiment should proceed. The instructor will guide the discussion. Technologies such as Clickers will be used to assess students' response. Using the virtual classroom environment, the instructor then demonstrates the experiment according to the results of the discussion and shows the result under different experiment conditions. This is again followed by an in-classroom discussion, before moving on to the next sub-session. IVOL is designed to engage students, by actively involving students in the decision making process throughout the experiment and constantly providing intellectual stimuli. Students are required to submit a lab report in conventional format following the IVOL class. In addition, formative assessments (questionnaires) and summative assessments (quizzes) are performed after IVOL to evaluate the effectiveness of learning. 


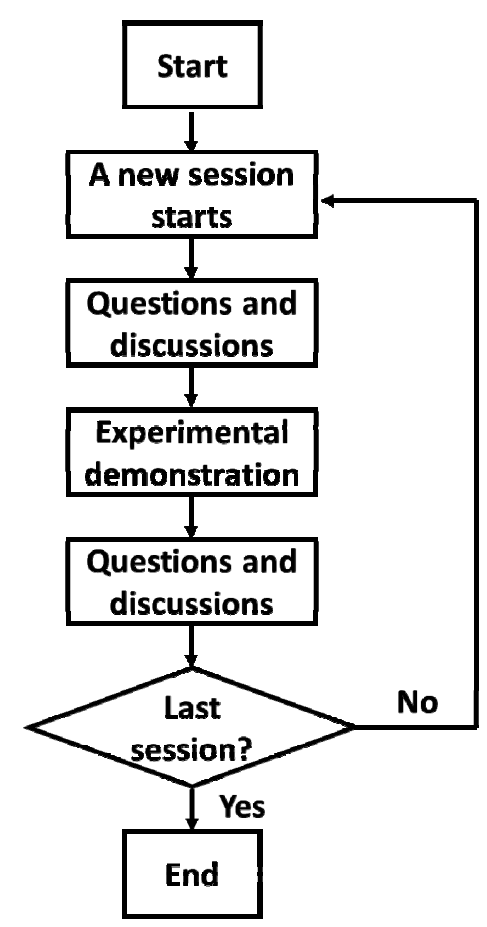

Figure. 2. IVOL flow chart.

\section{EXAMPLE OF IOVL CLASS: THE PRINCIPLE OF CONFOCAL MICROSCOPY}

In this study, we take the experiment to study the principle of confocal microscopy as an example of IOVL. Confocal microscopy is an optical imaging technique that provides cross-sectioning capability of tissue sample [5, 6]. The schematic of a confocal microscope is shown in Figure 3. A point light source illuminates the specimen. Light from the specimen is spatially filtered by a pinhole before detected. The confocal configuration, i.e., the use of point source and point detector that are in conjugation, enables depth resolved imaging. Assume light is reflected from a plane (Plane A in Figure 3) is in conjugation with the plane where the pinhole lies (Plane B in Figure 3). Such a geometry allows light reflected from plane A to be detected with high efficiency. In comparison, when light comes from a different plane, the reflected light will form an extended beam at Plane B where the pinhole lies. The pinhole rejects a large portion of the optical signal to be detected for out-of-focus photons, thus provides optical sectioning capability. In summary, a confocal microscope consists of a coherence light source for point illumination, imaging optics, detector, and a pinhole that is located at the plane in conjugation with the focal plane. 


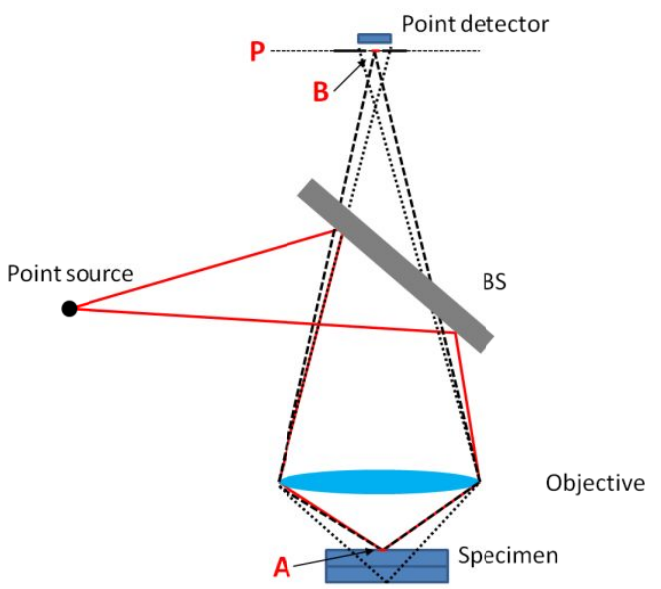

Figure. 3. Schematic diagram of a confocal microscope.

The modular design of the IOVL class to study the principle of confocal microscopy is illustrated in Figure 4.
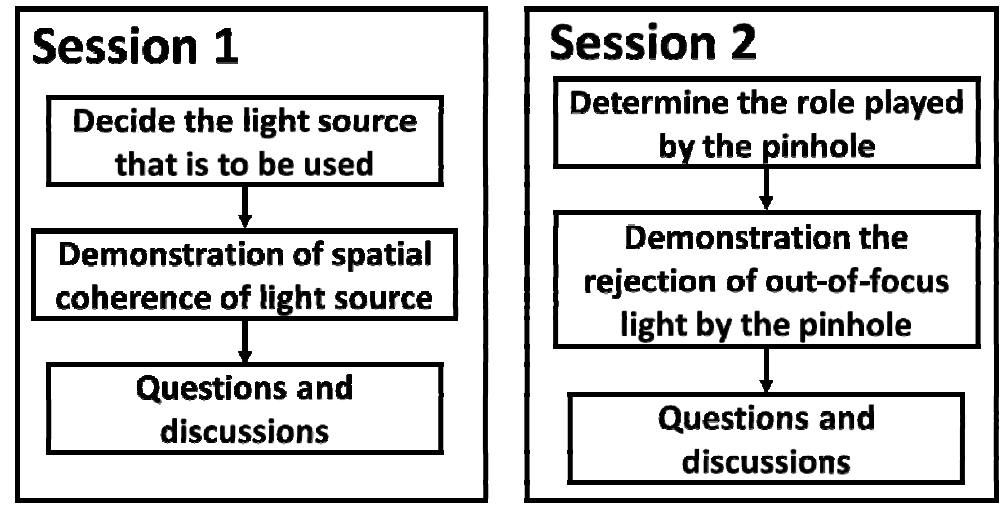

Figure. 4. Modular design of IOVL class to study the principle of confocal microscopy.

The first session of this experiment is to choose the appropriate light source for confocal microscopy. Students are provided with two different light sources. One is an incoherent LED, and the other is a coherent laser source. The concepts of spatial and temporal coherence are reviewed by the course instructor with multi-media assistance. Afterwards, the instructor asks the students to choose the light source and collects student response by Clickers or similar products. The instructor leads the discussion on the students' rationale for their specific choice. Afterwards, the instructor uses the virtual classroom environment to demonstrate the spatial coherence for different light sources. In the laboratory at a distance spatially or temporally, experimental demonstration of spatial coherence is provided. For example, the instructor in the lab places the light source at the back focal plane of a lens, and uses a screen to provide visualization of the light beam along the direction of light propagation. As shown in Figure 5, the light beam of the coherent laser source remains the same size after propagation for 4 (Figure 5 (a)), 8 (Figure 5 (b)) and 12 (Figure 5 (c)) inches. In comparison, the light beam of the incoherent LED source diverges after propagating for 4 (Figure 5 (c)), 8 (Figure 5 (d)) and 12 (Figure 5 (e)) inches. Results in Figure 5 suggest the beam from an incoherence light source cannot 
be collimated into parallel rays, or focused into a geometric point. After demonstration via virtual classroom environment, the instructor will lead additional discussion in the classroom, asks the students to choose the ideal light source for confocal microscopy, and then proceeds to the next session of the experiment.

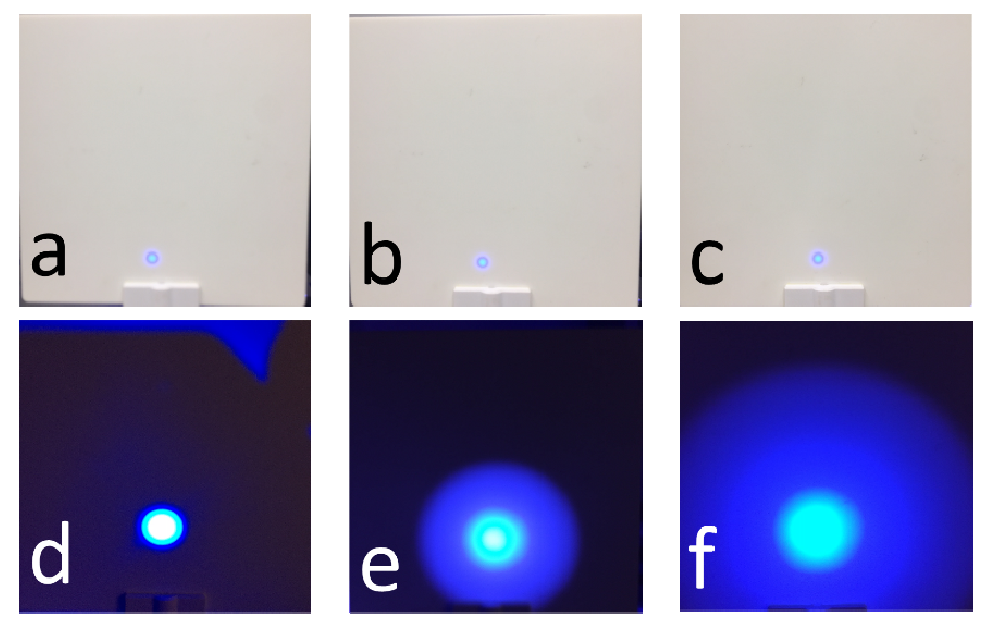

Figure. 5. Light beam after propagation. (a) collimated laser beam after propagation for 4 inches; (b) collimated laser beam after propagation for 8 inches; (c) collimated laser beam after propagation for 12 inches; (c) "collimated" LED beam after propagation for 4 inches; (d) "collimated" LED beam after propagation for 8 inches; (e) "collimated" LED beam after propagation for 12 inches.

The second session of this experiment is to investigate the role played by the pinhole in confocal imaging. The instructor will explain the configuration shown in Figure 3. Similar to the first session, the instructor discusses the about the system design and asks the students about the location to place the pinhole. Students' rationale for their specific choice of the pinhole location will lead the discussion on different phenomena when optical signal is detected with or without the pinhole as a spatial filter. Afterwards, experimental demonstration of confocal detection of optical signal is provided through the virtual classroom environment. Experiments will be carried out on the system shown in Figure 6 where a fiber connected to a photon detector serves as the pinhole detector. In the experimental demonstration, a mirror is used as the sample that is scanned axially by a mechanical actuator. The intensity of light is collected and observed as a function of time on the screen of an oscilloscope (Figure 7). Figure 7 (a) shows the variation of light intensity over time as the mirror is scanned across the plane in conjugation with the pinhole. Figure 7 (b) shows the variation of light intensity over time as the mirror is scanned within the same range with the pinhole removed. Clearly, the existence of the pinhole enables depth resolved detection of light. After experimental demonstration, the students are encouraged to comment on the phenomena observed, explain the underlying mechanism of confocal detection of optical signal, and come up with strategies for 3D optical imaging based on confocal principle. 


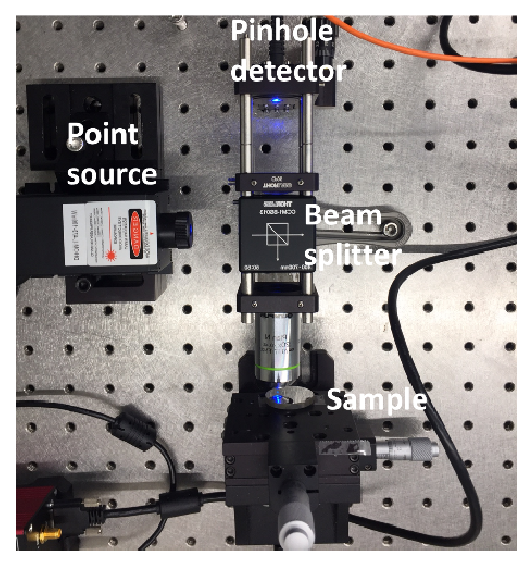

Figure. 6. Experimental setup for the demonstration of the principle of confocal microscopy
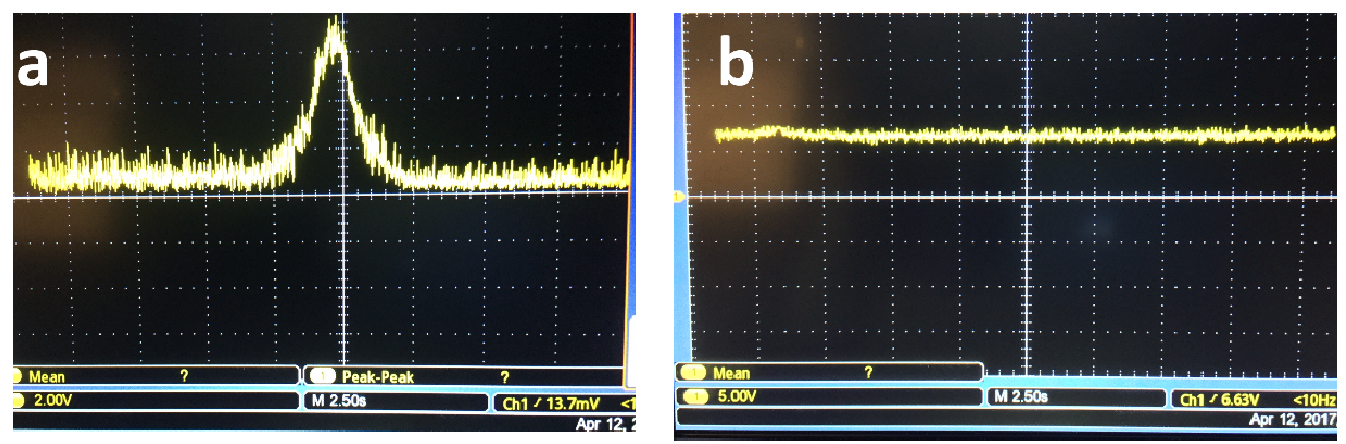

Figure. 7. (a) Light intensity captured when the sample mirror is scanned across the plane in conjugate with the pinhole; (b) light intensity captured when the sample mirror is scanned along the light propagation direction without the pinhole before photon detector.

\section{DISCUSSION AND CONCLUSION}

Unlike online lectures where efficacy can be evaluated through quizzes and write-ups, virtual labs requires an immersive and interactive experience for students to fully understand the concepts and predict outcomes. Furthermore, a coherent and intuitive laboratory procedure is required to minimize confusion for the students. We first presented an IVOL process that involves student participation and systematically evaluates students' progress. During each step of the process, students' input is taken as a guide to the next procedure. Therefore, students' decision will affect the outcome of the experiment. After each step, a discussion is carried out to ensure students understand the causal relationship between the decisions and outcomes. Two optical experiment demo is based on the confocal microscopy experiment is presented, which focuses on acquiring the depth information through a pin hole. In the first demo, students have the opportunity of deciding on the right light source. Depending on their choices, two different results are presented through IVOL. Through discussion, the causal relationship between light source and resolution is clarified. In the second experiment demo, students discuss the concept of using a pinhole to resolve depth information. Then they are given the choice to 
place the pinhole at multiple locations. As shown in Fig. 7b, without the pinhole before the detector, the detector will simply detect a baseline signal that carries no depth information. When the students decide to place the pinhole before the detector and move the pinhole for a small distance, they will observe the variation of signal intensity, which correlates with depth information. This interactive process ensures that students fully understand the principle of the confocal microscopy and its structure design. A systematic research investigating the efficacy of the IVOL is necessary and will be carried out.

The interactive virtual optical laboratory presents an online platform for students without the proper photonics lab equipment. In order to create an immersive experience, the system model focuses on facilitating discussion and students' decision making throughout the experiment. This interactive approach intends to mimic the real world hands on lab experience and decision making, which is essential for students to understand abstract concepts. We demonstrated two IVOL based optical experiment examples and addressed how the procedures ensure student leaning in this virtual environment. Future work will focus on expanding the IVOL platform into more optical experiments and systematically quantify its efficacy.

\section{REFERENCES}

[1] M. G. Moore, "Theory of transactional distance," Theoretical principles of distance education 1, 22-38 (1993).

[2] J. L. McBrien, R. Cheng, and P. Jones, "Virtual spaces: Employing a synchronous online classroom to facilitate student engagement in online learning," The International Review of Research in Open and Distributed Learning 10(2009).

[3] Z. Yang and Q. Liu, "Research and development of web-based virtual online classroom," Computers \& education 48, 171-184 (2007).

[4] B. Dalgarno, A. G. Bishop, W. Adlong, and D. R. Bedgood, "Effectiveness of a virtual laboratory as a preparatory resource for distance education chemistry students," Computers \& Education 53, 853-865 (2009).

[5] L. V. Wang and H.-i. Wu, Biomedical optics: principles and imaging (John Wiley \& Sons, 2012).

[6] J. Mertz, Introduction to optical microscopy (CSIRO, 2010), Vol. 138.

Proc. of SPIE Vol. 10452 104521D-7 